\title{
ANÁLISE DA FORÇA SUSTENTADA DE PREENSÃO PALMAR EM HOMENS E MULHERES POR MEIO DE DINAMÔMETRO ELETRÔNICO
}

\author{
N. N. ZAGO*, E. D. ZANON**, B. G. KIMURA** e L. F. R. M. FERNANDES*** \\ * Discente do Programa de Pós-Graduação em Educação Física, Universidade Federal do Triângulo \\ Mineiro, Uberaba, Brasil \\ ** Graduando em Fisioterapia, Universidade Federal do Triângulo Mineiro, Uberaba, Brasil \\ *** Docente do Departamento de Fisioterapia Aplicada, Universidade Federal do Triângulo \\ Mineiro, Uberaba, Brasil \\ e-mail: najarazago@hotmail.com
}

\begin{abstract}
Resumo: Em pacientes com traumas do punho e da mão, a análise da força sustentada de preensão palmar permite programar os exercícios isométricos e monitorar o progresso da força muscular durante o programa de reabilitação. Previamente a realização desta avaliação em pacientes com traumas do punho e da mão, foi realizado um estudo em uma amostra de indivíduos jovens, sem história de traumas nos membros superiores. Nesse sentido, esse estudo teve como objetivo verificar em que momento da isometria começa a diminuição da força comparando os lados dominante e não dominante de homens e mulheres. Foram recrutados 36 voluntários, sendo 17 homens e 19 mulheres com idade média de $22( \pm 2,0)$ anos. A força sustentada de preensão palmar foi mensurada durante 30 segundos utilizando um dinamômetro de preensão modelo G200, com célula de carga de precisão, da marca Biometrics ${ }^{\circledR}$. Observou-se que ocorreu um declínio da força sustentada de preensão palmar em ambos os membros e grupos, porém houve diferença estatística em relação aos primeiros 10 segundos e os últimos 10 segundos no membro dominante e não dominante dos homens e não dominante das mulheres. Conclui-se que houve uma diminuição da força de preensão palmar entre os primeiros 10 segundos e os últimos 10 segundo no membro dominante e não dominante para os homens, e somente para o membro não dominante para as mulheres.
\end{abstract}

Palavras-chave: Força Muscular, Contração Isométrica, Exercício Isométrico, Reabilitação, Fisioterapia.

\footnotetext{
Abstract: In patients with trauma of the wrist and hand, the analysis of sustained handgrip strength is an important item to program the isometric exercises and monitor the progress of muscle strength during the rehabilitation program. A sample of young individuals without trauma of upper limbs was used to conduction this test prior to patients with trauma of the wrist and hand. Thus, this study aimed to verify at what time of isometrics begins to decrease in strength comparing the dominant and non-dominant sides and with men and women. They recruited 36 volunteers, 17 men and 19 women with an average age of $22( \pm 2.0)$ years. Were
}

divided into two groups, men group (G1) and women group (G2), the volunteers who agreed to participate in this research. Sustained grip strength was measured for 30 seconds using a handgrip dynamometer model G200 with precision load cell, the Biometrics ${ }^{\circledR}$ brand. In the results was founded a decline in sustained grip strength in both members and groups, but there was a statistical difference from the first 10 seconds and the last 10 seconds in the dominant and non-dominant for men and not dominant for women. Concluded that there was a decrease in grip strength between the first 10 seconds and the last 10 seconds in the dominant and nondominant for men and only to the non-dominant limb for women.

Keywords: Muscle Strength, Isometric Contraction, Isometric Exercise, Rehabilitation, Physical Therapy Specialty.

\section{Introdução}

A força sustentada de preensão palmar é definida como uma medida de força exercida pela mão durante uma contração isométrica voluntária ao longo de um determinado tempo. Esse teste pode ser utilizado para diagnosticar doenças, programar os exercícios e monitorar o progresso da força muscular durante o programa de reabilitação [1].

Em pacientes com traumas do punho e da mão, essa avaliação permite quantificar o tempo de isometria para realização dos exercícios de fortalecimento. Para esses pacientes, a realização dos exercícios isométricos é importante para o fortalecimento dos músculos intrínsecos e extrínsecos da mão, porém a realização inadequada destes exercícios pode influenciar negativamente no ganho de força e no risco de desenvolver novas lesões musculares [2].

Nesse contexto, os exercícios isométricos são um grande aliado para o condicionamento físico, ganho de força e para reabilitação fisioterapêutica. A relação entre a carga da resistência isométrica e o tempo de manutenção da isometria durante a realização de exercícios é um fator importante para a prescrição dos exercícios [3], pois quanto maior o tempo de isometria 
maior será a redução da capacidade do músculo em gerar força ou realizar trabalho [2].

Em vista disso, torna-se importante a realização da avaliação da força sustentada de preensão palmar objetivando avaliar a condição física, muscular e de resistência previamente a realização de protocolos de fortalecimento isométrico. Buscando auxiliar o fisioterapeuta na escolha de protocolos adequados para fortalecimento da musculatura do punho e da mão, esse estudo teve como objetivo verificar se o tempo de isometria durante o teste de força sustentada de preensão palmar por 30 segundos pode levar a diminuição da força nos membros dominante e não dominante de homens e mulheres.

\section{Materiais e métodos}

Esta pesquisa caracteriza-se por ser um estudo transversal, composto por 36 voluntários jovens, sendo 17 homens e 19 mulheres com idade média de $22( \pm 2,0)$ anos, a maioria destros $(91,7 \%)$. A Tabela 1 apresenta um resumo das características dos voluntários. Os critérios de inclusão foram: a) ambos os sexos, b) idade entre 18 e 30 anos, c) que não realizem exercício físico para os membros superiores nos últimos 6 meses, d) ausência de doenças cardiovasculares e e) ausência doenças neuromusculares e musculoesqueléticas nos membros superiores.

Todos os voluntários foram informados sobre os objetivos e procedimentos da pesquisa e assinaram um Termo de Consentimento Livre e Esclarecido, conforme resolução nº66/12 do Conselho Nacional de Saúde (CNS). A pesquisa foi aprovada pelo Comitê de Ética em Pesquisa da Universidade Federal do Triângulo Mineiro, com parecer 2416.

Tabela 1: Características da amostra.

\begin{tabular}{lc}
\hline Características & $\begin{array}{c}\text { Média (DP) } \\
\text { ou n (\%) }\end{array}$ \\
\hline Gênero, n (\%) & $17(47,2)$ \\
Masculino & $19(52,8)$ \\
Feminino & $22( \pm 2)$ \\
Média de Idade (DP) & $22( \pm 2)$ \\
Homens & $33(91,7)$ \\
Mulheres & $2(5,5)$ \\
Dominância, n (\%) & $1(2,8)$ \\
Direita & \\
Esquerda & \\
Ambidestro & número da amostra; \% $=$ \\
\hline DP $=$ desvio padrão; n $=$ nón \\
porcentagem.
\end{tabular}

A força sustentada de preensão palmar foi mensurada utilizando o Kit E-link da marca Biometrics ${ }^{\circledR}$ modelo H500 - Hand kit que inclui um dinamômetro modelo G200 com célula de carga de precisão e software de avaliação para os membros superiores (EWS - Upper Extremity Evaluation Software). Foi utilizada uma taxa de amostragem de $20 \mathrm{~Hz}$ para coleta do teste.

A avaliação da força sustentada de preensão palmar foi realizada uma única vez em ambos os membros, alternadamente, e calculadas as médias totais das forças nos 30 segundos e a média das forças nos primeiros 10 segundos, de 10 a 20 segundos e de 20 a 30 segundos do teste (Figura 1).

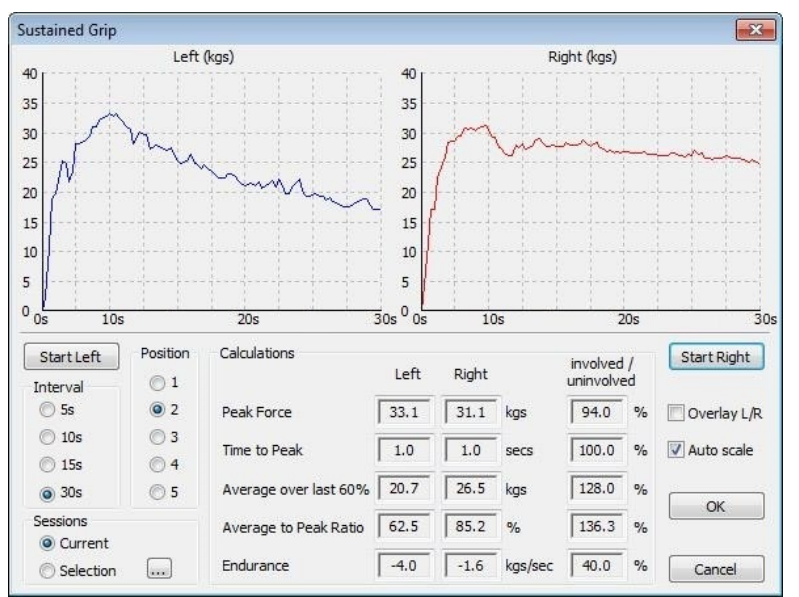

Figura 1: Representação de uma coleta realizada para análise da média da força sustentada de preensão palmar durante os 30 segundos.

Durante estas avaliações os voluntários permaneceram sentados, com o cotovelo fletido a $90^{\circ}$, antebraço e punho em neutro, segundo recomendação da Sociedade Americana dos Terapeutas da Mão [5,6]. Foi utilizada a empunhadura do dinamômetro na posição 3 para os homens, e 2 para mulheres [7].

Análise Estatística - Os dados das forças sustentada de preensão palmar foram processados e analisados por uma rotina no programa Matlab ${ }^{\circledR}$ (The MathWorks Inc., USA) versão R2015a. Em seguida foram submetidos a análise descritiva e inferencial, com nível de significância de 5\%. Na análise estatística descritiva, utilizou-se a média e o desvio padrão. Na estatística inferencial, para verificação da normalidade, utilizou-se o teste de Shapiro-Wilk. Para as análises foi utilizado ANOVA Multifatorial com post hoc de Tukey. Os dados foram processados por meio do programa Statistical Package for the Social Sciences (SPSS $®)$ versão 20.

\section{Resultados}

Nesse estudo foram analisados a força sustentada de preensão palmar durante 30 segundos de ambos os membros (dominante e não dominante) de homens e mulheres. A média e o desvio padrão das forças sustentada de preensão palmar durante os 30 segundos, nos membros dominante e não dominante de homens e 
mulheres foram representadas na Tabela 2 .

Tabela 2: Média e desvio padrão das forças sustentada de preensão palmar durante os 30 segundos, nos membros dominante e não dominante de homens e mulheres.

\begin{tabular}{llll}
\hline Grupo & Tempo & $\begin{array}{c}\text { Lado } \\
\text { Dominante } \\
\text { (Kgf) }\end{array}$ & $\begin{array}{c}\text { Lado Não } \\
\text { Dominante } \\
\text { (Kgf) }\end{array}$ \\
\hline G1 & 0 a 10s & $37,32( \pm 8,66)$ & $34,58( \pm 9,99)$ \\
& 10 a 20s & $32,23( \pm 7,36)$ & $28,64( \pm 8,80)$ \\
G2 & 20 a 30s & $27,94( \pm 7,20)$ & $26,54( \pm 7,15)$ \\
& 10 10s & $18,58( \pm 4,94)$ & $16,84( \pm 4,26)$ \\
& 20 a 30s & $14,37( \pm 4,08)$ & $13,25( \pm 3,0)$ \\
& & $13,70( \pm 3,75)$ & $12,07( \pm 3,04)$ \\
\hline
\end{tabular}

Kgf = quilograma força; G1 = grupo de homens; G2 = grupo de mulheres.

Os resultados obtidos nesse estudo, demonstraram que houve um declínio da força de preensão nos membros dominante e não dominante, em ambos os grupos (Figura 2 e 3).

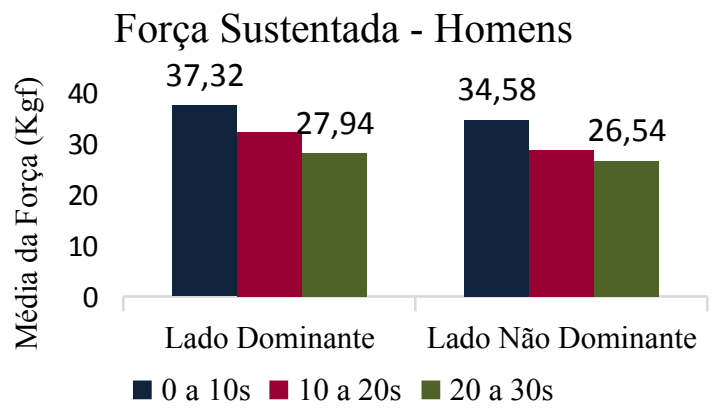

Figura 2: Representação gráfica das médias e desvios padrões das forças sustentada de preensão palmar nos primeiros 10 segundos, de 10 a 20 segundos e de 20 a 30 segundos, nos membros dominante e não dominante de homens.

$\mathrm{Kgf}=$ quilograma força; $\mathrm{s}=$ segundos.

\section{Força Sustentada - Mulheres}

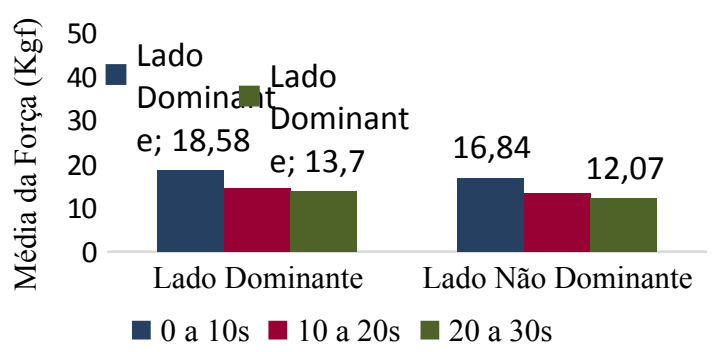

Figura 3: Representação gráfica das médias e desvios padrões das forças sustentada nos primeiros 10 segundos, de 10 a 20 segundos e de 20 a 30 segundos, nos membros dominante e não dominante de mulheres. $\mathrm{Kgf}=$ quilograma força; $\mathrm{s}=$ segundos.
A ANOVA foi utilizada para comparar a média das forças sustentadas de preensão nos primeiros 10 segundos, de 10 a 20 segundos e de 20 a 30 segundos do teste. No G1 foram encontradas diferenças significativas para a força sustentada de preensão palmar nos primeiros 10 segundos e para os últimos 10 segundos para os membros dominante e não dominante. Para o G2 foram encontradas diferenças significativas para a força sustentada de preensão palmar nos primeiros 10 segundos e para os últimos 10 segundos para não dominante.

\section{Discussão}

Os dinamômetros eletrônicos oferecem vantagens quando comparados aos dinamômetros hidráulicos, que incluem a análise da força durante uma contração isométrica prolongada, o aumento da sensibilidade aplicada as forças, as leituras digitais aumentam a confiabilidade inter e intra avaliadores e promovem uma redução dos erros manuais de leitura e do cálculo das médias das forças $[8,9]$.

Esses equipamentos apresentam uma maior precisão dos dados através do tempo, além de fornecer um feedback visual e auditivo importante no incentivo durante os testes de contração isométrica [10].

Nesse estudo, o dinamômetro eletrônico foi utilizado para verificar se o tempo de isometria durante o teste de força sustentada de preensão palmar por 30 segundos pode levar a diminuição da força nos membros dominante e não dominante de homens e mulheres.

Os resultados encontrados demonstraram que houve um declínio da força sustentada de preensão palmar nos membros dominante e não dominante, em ambos os grupos. Esse declínio foi maior em relação aos primeiros 10 segundos e os últimos 10 segundos do teste.

Vários fatores podem ter influenciado na produção de força da mão, tais como: variações no comprimento muscular, tipo de fibra, flexibilidade dos músculos e do tendão, condições articulares, tipo de preensão, largura do objeto de preensão e a configuração do corpo/articular [11].

Nesse sentido, para a realização da prática de exercícios isométricos é necessário o conhecimento prévio sobre o estado físico geral dos indivíduos, para quantificar o tempo máximo da isometria. É de extrema importância a orientação, acompanhamento e prescrição de exercícios de forma correta e bem orientada pelos profissionais responsáveis, uma vez que pode refletir negativamente na segurança, bem-estar físico e muscular.

Com isso, verifica-se a necessidade da realização do teste de força sustentada de preensão palmar previamente a prescrição dos exercícios isométricos. Isso é importante para garantir que os indivíduos recebam um tratamento adequado e individualizado.

Para Kisner (2005) os exercícios isométricos, quando utilizados para o fortalecimento, devem ser realizados com uma duração de 6 a 10 segundos. Porém 
para esse estudo não foram encontradas diferenças significativas da força sustentada de preensão palmar nos intervalos de 0 a 10 segundos e de 10 a 20 segundos do teste, sugerindo que para o fortalecimento dos músculos intrínsecos e extrínsecos da mão, o tempo de isometria pode ser maior, atingindo até 20 segundos de isometria, em indivíduos jovens sem lesão ou traumas do punho e da mão.

Outros estudos posteriormente serão realizados para estudar mais precisamente o tempo de isometria indicado para a realização do fortalecimento dos músculos intrínsecos e extrínsecos da mão em indivíduos jovens, dentro do intervalo de 10 a 20 segundos.

\section{Conclusão}

Conclui-se que houve uma diminuição da força de preensão palmar entre os primeiros 10 segundos e os últimos 10 segundo no membro dominante e não dominante para os homens, e somente para o membro não dominante para as mulheres.

\section{Agradecimentos}

Os autores agradecem ao LABCOM por ceder espaço e equipamentos, a Coordenação de Aperfeiçoamento de Pessoal de Nível Superior (CAPES) e a Fundação de Amparo à Pesquisa de Minas Gerais (FAPEMIG) pelo apoio financeiro e aos voluntários que aceitaram participar, tornando possível a realização desse trabalho.

\section{Referências}

[1] ALLEN D, BARNETT F. Reliability and validity of an electronic dynamometer for measuring grip strength. Int J Ther Rehabil. 2011 May; 18(5):258-64.

[2] TROSSMAN PB, SULESKI KB, LI P-W. Test-retest reliability and day-to-day variability of an isometric grip strength test using the work simulator. OTJR Occup Particip Health. 1990;10(5):266-279.

[3] KISNER C. Exercícios terapêuticos: fundamentos e técnicas. 4th ed. Barueri (SP): Manole; 2005. 841 p.

[4] OLDFIELD RC. The assessment and analysis of handedness: the edinburgh inventory. Neuropsychologia. 1971;9:97-113.

[5] FESS EE. Grip Strength. 2nd ed. Chicago: American Society of Hand Therapists; 1992.

[6] FESS EE, MORAN CA. Clinical assessment recommendations. Philadelphia: American Society of Hand Therapists; 1981.

[7] FERNANDES LFRM, BERTONCELLO D, PINHEIRO NM, DRUMOND LC. Correlações entre força de preensão manual e variáveis antropométricas da mão de jovens adultos. Fisioter E Pesqui. 2011;18(2):151-156.

[8] SVENS B, LEE H. Intra-and inter-instrument reliability of Grip-Strength Measurements: GripTrack ${ }^{\mathrm{TM}}$ and Jamar ${ }^{\circledR}$ hand dynamometers. The British Journal of Hand Therapy. 2005;10(2):47-55.

[9] SHECHTMAN O, GESTEWITZ L, KIMBLE C. Reliability and Validity of the DynEx Dynamometer. J Hand Ther. 2005 Jul;18(3):339-47.

[10] BATISTA J, WIBELINGER L, MARCHI A, SCHNEIDER R, PASQUALOTTI A. Reabilitação de idosos com alterações cognitivas através do videogame Nintendo Wii. RBCEH. 2012 maio-ago;9(2):292-9.

[11] LI ZM. The influence of wrist position on individual finger forces during forceful grip. J Hand Surg. 2002;27(5):886-896. 